\title{
The no-wall holographic model for vector quarkonia
}

\author{
Sergey Afonin ${ }^{1, \star}$ and Ilya Pusenkov ${ }^{1, \star \star}$ \\ ${ }^{1}$ Saint Petersburg State University, 7/9 Universitetskaya nab., St.Petersburg, 199034, Russia
}

\begin{abstract}
We use the no-wall holographic approach (a relative of the soft-wall one) to construct a universal description of vector mesons with arbitrary quark masses. The proposed model predicts a specific dependence of the parameters of radial Regge trajectories on the quark masses in a reasonable agreement with the meson phenomenology and some theoretical expectations.
\end{abstract}

The bottom-up holographic approach to QCD introduced in Ref. [1] has many interesting applications in the phenomenology of the strong interactions. Usually this approach is applied to the spectroscopy of light hadrons and the related physics [2]. The heavy-quark sector is addressed relatively seldom (see, e.g., [3, 4] and references therein). In this sector, only the unflavored vector mesons have a rich established radial spectrum [5] that can be compared with theoretical predictions. Our aim is a description of this spectrum within the no-wall holographic approach [6].

The action of the simplest holographic no-wall model is defined by

$$
S=\int d^{4} x d z \sqrt{g}\left\{\sum_{i}\left(\left|D X_{i}\right|^{2}-m_{i}^{2}\left|X_{i}\right|^{2}\right)-\frac{1}{4 g_{5}^{2}} F_{M N}^{2}\right\},
$$

where

$$
g=\left|\operatorname{det} g_{M N}\right|, \quad F_{M N}=\partial_{M} V_{N}-\partial_{N} V_{M}, \quad D_{M} X_{i}=\partial_{M} X_{i}-i V_{M} X_{i},
$$

and $M, N=0,1,2,3,4$. The action (1) is defined in the $\mathrm{AdS}_{5}$ space with the metrics

$$
g_{M N} d x^{M} d x^{N}=\frac{R^{2}}{z^{2}}\left(\eta_{\mu \nu} d x^{\mu} d x^{\nu}-d z^{2}\right) .
$$

Here $\eta_{\mu v}=\operatorname{diag}(1,-1,-1,-1)$ and $R$ denotes the $\mathrm{AdS}_{5}$ radius. Below we set $R=1$ for simplicity. The holographic coordinate $z \geq 0$ has the interpretation of inverse energy scale. The UV boundary $z=0$ represents the 4D Minkovski space. The 5D vector field $V_{M}(x, z)$ is dual to the $4 \mathrm{D}$ conserved vector current $j_{\mu}=\bar{\psi}_{q} \gamma_{\mu} \psi_{q}$ for any quark flavor $q$. The gauge invariance of the action (1) allows to choose a convenient axial gauge $V_{z}=0$. On the UV boundary, the scalar fields $X_{i}$ are identified with sources of various QCD operators with canonical dimension $i$. The corresponding 5D masses are [7]

$$
m_{i}^{2}=i(i-4)
$$

${ }^{\star}$ e-mail: s.afonin@spbu.ru

$\star \star$ e-mail: i.pusenkov@spbu.ru 
By assumption, the fields $X_{i}$ acquire $z$-dependent vacuum expectation values $\left\langle X_{i}\right\rangle$ which represent the $x$-independent solutions of the equation of motion,

$$
\partial_{z}\left(\frac{1}{z^{3}} \partial_{z} X_{i}\right)=\frac{m_{i}^{2}}{z^{5}} X_{i}
$$

with the UV boundary condition

$$
\left.\left\langle X_{i}\right\rangle\right|_{z=0}=0 .
$$

The vector physical modes are defined by the plane-wave ansatz $V_{\mu}(x, z)=e^{i p x} v(z) \varepsilon_{\mu}$. The mass spectrum of these modes is given by the eigenvalues $M_{n}^{2}=p_{n}^{2}$ of the corresponding equation of motion,

$$
\partial_{z}\left(\frac{1}{z} \partial_{z} v_{n}\right)+\frac{M_{n}^{2}}{z} v_{n}=\frac{2 g_{5}^{2}}{z^{3}} v_{n} \sum_{i}\left\langle X_{i}\right\rangle^{2} .
$$

The change of variables $v_{n}=\sqrt{z} \psi_{n}$ brings this equation into the Schrödinger form

$$
-\partial_{z}^{2} \psi_{n}+\left(\frac{3}{4 z^{2}}+2 g_{5}^{2} f(z)\right) \psi_{n}=M_{n}^{2} \psi_{n},
$$

where

$$
f(z)=\frac{1}{z^{2}} \sum_{i}\left\langle X_{i}\right\rangle^{2}
$$

determines the analogue of potential in the Schrödinger equation.

Let us consider the dimension-two operator, $i=2$, and neglect all others. The solution of Eq. (5) satisfying (6) reads

$$
\left\langle X_{2}\right\rangle=C_{2}^{(1)} z^{2}+C_{2}^{(2)} z^{2} \ln z .
$$

Choosing $C_{2}^{(2)}=0$, the equation (8) coincide with the equation on the mass spectrum in the standard holographic soft-wall model [8].

The potential in Eq. (8) is written near the UV boundary, where, by assumption, the holographic correspondence allows to use the QCD inputs. In order to calculate the mass spectrum we must continue the function $f(z)$ to the infrared domain, $z \rightarrow \infty$. The linear radial Regge spectrum observed in the unflavored light and heavy quarkonia [9] can be obtained only if

$$
\left.f(z)\right|_{z \rightarrow \infty}=a^{2} z^{2} .
$$

The UV asymptotics of the 5D field dual to the dim 2 operator provides the correct IR asymptotics automatically.

The dependence of the linear mass spectrum on the quark mass appears from the v.e.v. $\left\langle X_{3}\right\rangle$ as the field $X_{3}$ is dual to the quark bilinear operator $\bar{q} q$. The quark mass emerges from the following AdS/CFT prescription: The solution of classical e.o.m. for a scalar field $\Phi$ corresponding to an operator $O$ of canonical dimension $i$ near the 4D boundary $z \rightarrow 0$ has the form [10]

$$
\Phi(x, z) \rightarrow z^{4-i}\left[\Phi_{0}(x)+O\left(z^{2}\right)\right]+z^{i}\left[\frac{\langle O(x)\rangle}{2 i-4}+O\left(z^{2}\right)\right],
$$

where $\Phi_{0}(x)$ acts as a source for $O(x)$ with the v.e.v. $\langle O(x)\rangle$. In the real QCD, the quark mass $m$ acts as the source for the operator $\bar{q} q$. 
For the canonical dimension $i=3$, the solution of Eq. (5) satisfying (6) is $\left\langle X_{3}\right\rangle=C_{3}^{(1)} z+C_{3}^{(2)} z^{3}$. According to the prescription (12), this solution can be rewritten in terms of the physical quantities,

$$
\left\langle X_{3}\right\rangle=\xi m z+\frac{\sigma}{2 \xi} z^{3},
$$

where $\sigma$ stays for the quark condensate $\langle\bar{q} q\rangle$ and the normalization factor $\xi$ is [11],

$$
\xi^{2}=\frac{N_{c}}{4 \pi^{2}}
$$

The solution (13) allows to extract explicitly the $m$-dependent terms in (9),

$$
f(z) \rightarrow \xi^{2} m^{2}+m \sigma z^{2}+\tilde{f}(z)
$$

where the contribution $\frac{\sigma^{2}}{4 \xi^{2}} z^{6}$ is absorbed into the new sum $\tilde{f}(z)$. The Regge form of the spectrum appears in the case of the asymptotics

$$
\left.\tilde{f}(z)\right|_{z \rightarrow \infty}=a^{2} z^{2}+\delta,
$$

which will be considered as our choice of $\tilde{f}(z)$.

We arrive then at the following equation on the mass spectrum,

$$
-\partial_{z}^{2} \psi_{n}+\left[\frac{3}{4 z^{2}}+2 g_{5}^{2}\left(\sigma m+a^{2}\right) z^{2}+2 g_{5}^{2} \xi^{2} m^{2}+2 g_{5}^{2} \delta\right] \psi_{n}=M_{n}^{2} \psi_{n} .
$$

The spectrum of Eq. (17) is

$$
M_{n}^{2}=4 \sqrt{2} g_{5} \sqrt{\sigma m+a^{2}}(n+1)+2 g_{5}^{2} \xi^{2} m^{2}+2 g_{5}^{2} \delta .
$$

This expression demonstrates the parametric dependence of the linear spectrum on the quark mass $m$. The spectrum (18) can be written in a more compact form,

$$
M_{n}^{2}=\sqrt{\alpha+\beta m}(n+1)+\gamma m^{2}+\delta .
$$

The value of constant $\gamma$ follows from the relations (14) and the normalization factor for the 5D massless vector fields, $g_{5}^{2}=\frac{12 \pi^{2}}{N_{c}}[1]$,

$$
\gamma=2 g_{5}^{2} \xi^{2}=6
$$

The ensuing phenomenological fits are elaborated in Ref. [3]. It can be also shown [3] that the behavior of the obtained slope in the chiral limit, $m \rightarrow 0$, matches a prediction of the dual Veneziano-like amplitudes with the chiral symmetry breaking [12].

The spectrum (19) predicts that the binding energy grows linearly with the quark mass in the heavy quark limit, $m \rightarrow \infty$, due to the relation (20) showing that $\gamma>4$. This behavior seems to agree with the experimental data [5]. For instance, one has for the ground states $M_{\psi}-2 m_{c} \approx 0.56 \mathrm{GeV}$ and $M_{\Upsilon}-2 m_{b} \approx 1.1 \mathrm{GeV}$ if $m_{c, b}$ are taken at $2 \mathrm{GeV}$ (taking $m_{b}$ at $5 \mathrm{GeV}$ leads to $M_{\Upsilon}-2 m_{b} \approx 2.5 \mathrm{GeV}$ ). Such a behavior admits a simple interpretation: When a non-relativistic quark of mass $m$ is created and moves with the velocity $v$, the binding energy must compensate its kinetic energy $\frac{m v^{2}}{2}$.

It should be emphasized that the mass relation of the kind (18) emerges within a whole class of holographic models and we have presented a particular model from this class. An example of another model of this sort is constructed in Ref. [3].

The proposed approach can be applied to various physical problems including the hadron spectroscopy, finite-temperature and density effects, hadron formfactors etc.

Acknowledgments. The work was supported by the Saint Petersburg State University research grant 11.38.189.2014 and by the RFBR grant 16-02-00348-a. 


\section{References}

[1] J. Erlich, E. Katz, D. T. Son and M. A. Stephanov, Phys. Rev. Lett. 95, 261602 (2005); L. Da Rold and A. Pomarol, Nucl. Phys. B 721, 79 (2005).

[2] J. Erlich, Contemp. Phys. 56, 159 (2015) [arXiv:1407.5002 [hep-ph]].

[3] S. S. Afonin and I. V. Pusenkov, Phys. Lett. B 726, 283 (2013).

[4] T. Gutsche, V. E. Lyubovitskij, I. Schmidt and A. Vega, Phys. Rev. D 87, 056001 (2013).

[5] K. A. Olive et al. (Particle Data Group), Chin. Phys. C 38, 090001 (2014).

[6] S. S. Afonin, Int. J. Mod. Phys. A 26, 3615 (2011); Phys. Lett. B 678, 477 (2009).

[7] E. Witten, Adv. Theor. Math. Phys. 2, 253 (1998); S. S. Gubser, I. R. Klebanov and A. M. Polyakov, Phys. Lett. B 428, 105 (1998).

[8] A. Karch, E. Katz, D. T. Son and M. A. Stephanov, Phys. Rev. D 74, 015005 (2006).

[9] S. S. Afonin and I. V. Pusenkov, Phys. Rev. D 90, 094020 (2014); Mod. Phys. Lett. A 29, 1450193 (2014).

[10] I. R. Klebanov and E. Witten, Nucl. Phys. B 556, 89 (1999).

[11] A. Cherman, T. D. Cohen and E. S. Werbos, Phys. Rev. C 79, 045203 (2009).

[12] M. Ademollo, G. Veneziano and S. Weinberg, Phys. Rev. Lett. 22, 83 (1969). 